\title{
School marks for Swedish children whose mothers had diabetes during pregnancy: a population-based study
}

\author{
G. Dahlquist • B. Källén
}

Received: 2 March 2007 / Accepted: 28 May 2007 /Published online: 7 July 2007

(C) Springer-Verlag 2007

\begin{abstract}
Aims/hypothesis To study, at a population level, school performance when leaving compulsory school of Swedish children whose mothers had diabetes during pregnancy compared with a reference population.

Methods We linked the Swedish Medical Birth Register with the Swedish School Mark Register, which contains school marks for all children in Sweden when leaving compulsory school. A total of 6,397 children were identified whose mothers had a diagnosis of diabetes during pregnancy in the years 1973 to 1986. Data on these children were compared with 1,300,683 children whose mothers had no diagnosis of diabetes during pregnancy. Risks were estimated as odd ratios (ORs) after adjustment for year of birth, maternal age, parity and educational level of the mother.

Results The children's average numerical school marks (for children leaving school between 1988 and 1997) were statistically significantly lower among children born to mothers with diabetes in pregnancy compared with reference children $(3.13 \pm 0.01$ vs $3.23, p<0.001)$. The effect was similar among boys and girls. There was also an effect of maternal diabetes during pregnancy on the risk of the child not completing compulsory school (OR 1.25; 95\% CI 1.101.43 , and after exclusion of infants with certain perinatal characteristics an OR of $1.25 ; 95 \%$ CI $1.02-1.53)$. When
\end{abstract}

G. Dahlquist $(\bowtie)$

Department of Clinical Sciences, Paediatrics, Umeå University, 90185 Umeå, Sweden

e-mail: gisela.dahlquist@pediatri.umu.se

B. Källén

Tornblad Institute, Lund University,

Lund, Sweden sports and the core subjects mathematics, English and Swedish were studied, there were increased risks of having scores below pass level and decreased probabilities of having scores above pass level for children of mothers with diabetes during pregnancy.

Conclusions/interpretation Children of mothers with diabetes during pregnancy performed slightly but significantly less well than reference children when leaving compulsory school at about 16 years old; this was also seen after adjustment for some putative perinatal and social confounders.

Keywords Children - Diabetes in pregnancy

School performance

Abbreviation
OR odds ratio

Introduction

A diagnosis of diabetes in the mother during pregnancy is associated with an increased risk for the fetus, including increased perinatal mortality, malformations, macrosomia and other neonatal complications. The occurrence of these perinatal complications has decreased considerably in many countries, possibly due to better metabolic control both before and during pregnancy [1]. Long-term effects of an intrauterine milieu complicated by maternal diabetes have also been described. As well as potential risk of type 2 diabetes and metabolic syndrome, adverse psychomotor development has been described $[1,2]$. Conflicting results have been reported concerning possible adverse events on intellectual function and attention deficits of children of diabetic mothers [3-7]. Such effects have been associated with markers of poor 
metabolic control [7]. Most of these studies are hospitalbased and include a limited number of cases.

In Sweden, a country with a population at high risk of both type 1 and type 2 diabetes, national healthcare programmes have focused on metabolic control of diabetes in pregnancy, and insulin pump or other intensive treatment regimens are recommended [8].

We have used nationwide population registers to study school performance, evaluated as school marks when leaving compulsory school (usually at 16 years of age). We compared children whose mother had diabetes in pregnancy with children whose mothers had no diabetes diagnosis during pregnancy and adjusted for some potential confounders.

\section{Methods}

\section{Ethical approval}

The study was approved by the regional research ethics committee in Umeå, which gives approval according to Swedish law on research ethics in line with the principles of the Declaration of Helsinki and the European Convention on Human Rights in Biomedicine and by the National Board of Health and Welfare.

\section{Registers used}

The Swedish School Mark Register records information on all school marks for children leaving Swedish compulsory schools [9]. When leaving compulsory school, the children are usually 16 years old. The Swedish Medical Birth Register $[10,11]$ contains data on pregnancy, delivery and the neonatal period for nearly all infants born in Sweden since 1973, with information on deaths and emigration linked. A linkage between the School Mark Register and the Medical Birth Register was performed and the highest final education of the mothers as recorded in the Swedish Educational Register (Statistics, Sweden) in 2002 was added. Individual identification was removed from the linked register. It was not possible to distinguish preexisting type 1 diabetes from type 2 or gestational diabetes, because before 1987 the classification code used in Sweden (ICD-8) did not differentiate the types.

From the Medical Birth Register, we identified children born of mothers with diabetes during pregnancy during the years 1973 to1986 and who left school during the years 1988 to 2003. A total of 6,397 children whose mothers had diabetes were identified and were compared with 1,300,683 children whose mothers had no diagnosis of diabetes. Since children of women with diabetes have an increased risk of childhood-onset diabetes, which in itself may affect their school performance, we linked the data also to the Swedish Childhood Diabetes Register [12] and identified 114 of the diabetic children whose mothers had diabetes during pregnancy.

\section{School mark system}

From 1988 to 1997 the school marks in Sweden were numerical on a five-level scale. From 1998 the marks became alphabetical with four levels: not passed, passed, passed with distinction, and passed with excellence. The marking systems are not comparable, since there are different marking systems and levels. In addition, while the numerical school marks were comparative and standardised using national standard achievement tests in mathematics, English and Swedish, the alphabetical marks were criterion-referenced, with each pupil expected to fulfil the criterion for the lowest level. Some children completed normal compulsory school but had no mark in some subjects. There are several reasons for this, for example that they had not attended class enough times.

\section{Analysis}

Children who died or emigrated before the age of 16 years were excluded. We made four separate comparisons.

Comparison $1 \mathrm{We}$ analysed the risk of a child not completing compulsory school. The reason for this can vary. Some children may have completed school abroad, for instance, but the majority of children who did not have data in the school mark register had gone to school outside the normal school system. Children who were diagnosed with a mental deficiency, severe hearing or vision problems attended special schools and possible school marks were not included in the school mark register.

In this analysis, we made a number of exclusions to evaluate the impact of some factors: preterm birth $(<37$ completed weeks), low Apgar score $(<7$ at $5 \mathrm{~min})$, twinning, macrosomia ( $>4,500 \mathrm{~g}$ birthweight), caesarean section and presence of a diagnosis of a congenital malformation in the Medical Birth Register. We also analysed the effect of exclusion of all these factors. We considered the importance of a child's own diabetes by exclusion of such children, identified from the Swedish Childhood Diabetes Register. Finally, we separately excluded all women born outside Sweden. We compared the outcome among boys and girls separately.

Comparison 2 We then analysed - for the years 1988-1997 when the numerical mark system was used - the mean mark for each child, based on all subjects where he/she had marks. That mean value was compared with the expected 
mean, estimated from the reference children born the same year, with the same maternal age and parity and maternal education; the difference was expressed as a $t$ value, calculated from the SD among the reference children in that level. The means of these $t$ values were compared with the expected value of 0 (if no difference existed between the two groups of children) with a new $t$ test, called $t^{*}$. In addition, these calculations were made separately for boys and girls.

Comparison $3 \mathrm{We}$ analysed the distribution of school marks within sports and the three core subjects: mathematics, Swedish and English. We then separately reported results for the period with numerical marks and for the period with alphabetical marks. Comparisons were made between children whose mothers had diabetes during pregnancy and children whose mothers had no diabetes diagnosis during pregnancy.

Comparison 4 For the period 1988 to 1997, children had a choice between a common and an advanced course in English and in mathematics. We studied the risk of choosing the common courses among children of women with diabetes vs other children.

\section{Statistical analysis}

The main tool for statistical analysis was the MantelHaenszel method with adjustments for four potential confounders: year of birth, maternal age, parity and maternal educational level. The risks were expressed as odds ratios (ORs) with 95\% CIs estimated by Miettinen's method. Comparisons between two stratified ORs were made as two-tailed $z$ tests based on the estimated variances.

\section{Results}

Not completing normal compulsory school

Table 1 shows the risk of a child whose mother had diabetes of having no school marks from compulsory school. There was a $25 \%$ increased risk of not having any school marks for children of mothers with diabetes during pregnancy compared with the other children, and this moderate risk increase was highly statistically significant.

Exclusions were then made for preterm birth $(<37$ weeks), low Apgar score $(<7)$, twinning, high birthweight $(>4,500 \mathrm{~g})$, delivery by caesarean section, or any malformation diagnosed. Each one of these exclusions had little effect on the OR and exclusion of all did not affect the OR, but of course widened the CI. The statistical significance was maintained.

When children with diabetes were excluded from the analysis (leaving for analysis 6,283 children born of women with diabetes) no effect was seen on the OR $(1.25 ; 95 \%$ CI 1.10-1.43). Exclusion of women born abroad increased the OR slightly (1.28; 95\% CI 1.12-1.47).

When analysing separately the effect by sex there was a tendency for a higher risk for boys (OR 1.32; 95\% CI 1.121.56) vs girls (OR $1.17 ; 95 \%$ CI $0.96-1.42$ ) but the difference between the sexes was not statistically significant $(z=0.90$, $p=0.27$ ).

\section{Mean numerical school marks}

For children who received marks on leaving compulsory school for the years 1988 to 1997 the global mean of numerical school marks was slightly but statistically significantly decreased for children of diabetic mothers (Table 2).

Table 1 Effect of diabetes during pregnancy on the risk of children not attending compulsory school

\begin{tabular}{|c|c|c|c|c|c|c|}
\hline \multirow[t]{2}{*}{ Group } & \multicolumn{2}{|c|}{ Children of diabetic mothers } & \multicolumn{2}{|c|}{ Children of non-diabetic mothers } & \multirow[t]{2}{*}{ OR } & \multirow[t]{2}{*}{$95 \% \mathrm{CI}$} \\
\hline & No marks & Marks & No marks & Marks & & \\
\hline All & 245 & 6,145 & 36,315 & $1,264,368$ & 1.25 & $1.10-1.42$ \\
\hline \multicolumn{7}{|l|}{ Exclusions } \\
\hline$<37$ weeks & 185 & 5,075 & 32,748 & $1,190,109$ & 1.14 & $0.96-1.25$ \\
\hline Apgar score $<7$ & 226 & 5,876 & 34,410 & $1,224,014$ & 1.17 & $1.01-1.37$ \\
\hline Twins & 243 & 6,016 & 35,486 & $1,243,258$ & 1.16 & $1.00-1.35$ \\
\hline $\begin{array}{c}\text { Birthweight } \\
>4,500 \mathrm{~g}\end{array}$ & 237 & 5,783 & 35,495 & $1,219,597$ & 1.18 & $1.01-1.37$ \\
\hline Caesarean & 152 & 3,886 & 31,509 & $1,131,399$ & 1.17 & $0.97-1.43$ \\
\hline Malformation & 226 & 5,795 & 32,490 & $1,196,599$ & 1.18 & $1.01-1.38$ \\
\hline All exclusions & 104 & 2,999 & 24,486 & 983,075 & 1.20 & $1.02-1.53$ \\
\hline
\end{tabular}

ORs with 95\% CIs after adjustment for year of birth, maternal age, parity and maternal education. Analysis was performed on all children followed by analyses after various exclusions. 
Table 2 The effect on numerical school marks of having a mother with diabetes during pregnancy: children leaving school in 1988-1997

\begin{tabular}{llllrr}
\hline & $n$ & Children of diabetic mothers & Children of non-diabetic mothers & Mean difference & $t^{*}$ \\
\hline All & 2,909 & $3.13 \pm 0.01$ & 3.23 & $-0.25 \pm 0.04$ & -6.4 \\
Boys & 1,484 & $2.98 \pm 0.02$ & 3.10 & $-0.25 \pm 0.04$ & -5.8 \\
Girls & 1,425 & $3.27 \pm 0.02$ & 3.39 & $-0.21 \pm 0.05$ & -4.5 \\
\hline
\end{tabular}

Means of school marks in all subjects are given, with those for children of diabetic mothers \pm SEM; mean difference represents the mean of $t$ values (with SEM), estimated for each child as the number of SDs from the reference population according to the year of birth, maternal age, parity and maternal educational level. The $t^{*}$ value compares this mean with zero and gives a $p$ value

School marks in four subjects

When looking at different levels of school marks we used sports, mathematics, Swedish and English language as examples. There was a slight increase of risk of not passing in all subjects for children of mothers with diabetes during pregnancy and a decrease in probability of achieving a mark of 'passed with distinction or excellence' (Table 3). The increase was, however, not statistically significant for the numerical marks (1988-1997) for the Swedish and English language. When looking for sex effects no specific pattern appeared (data not shown). Figure 1 shows for the four subjects studied ORs and $95 \%$ CIs for each of the numerical or alphabetical marks.

\section{Choice of common or advanced course}

During the years 1988 to 1997 , it was possible for the children to choose between courses with two levels of mathematics and English, common or advanced. Children whose mothers had diabetes during pregnancy had a significantly higher attendance at the less advanced course in both mathematics and English language (Table 4). This effect was similar for boys and girls.

Table 3 Effect of having a mother with diabetes in pregnancy on school marks in sports and three core subjects when leaving compulsory school

\begin{tabular}{|c|c|c|c|c|c|c|}
\hline \multirow[t]{3}{*}{ School marks } & \multicolumn{6}{|c|}{ Year of children leaving compulsory school } \\
\hline & \multicolumn{3}{|c|}{ 1988-1997 } & \multicolumn{3}{|c|}{$1998-2003$} \\
\hline & $n$ & OR & $95 \% \mathrm{CI}$ & $n$ & OR & $95 \% \mathrm{CI}$ \\
\hline \multicolumn{7}{|l|}{ Sports } \\
\hline No marks & 74 & 1.11 & $0.87-1.40$ & 53 & 1.06 & $0.80-1.40$ \\
\hline Not passed & 546 & 1.19 & $1.08-1.32$ & 281 & 1.21 & $1.06-1.38$ \\
\hline Passed & 1,408 & 1.00 & Reference group & 1,338 & 1.00 & Reference group \\
\hline Passed with distinction or excellence & 903 & 0.69 & $0.64-0.75$ & 1,542 & 0.62 & $0.55-0.69$ \\
\hline \multicolumn{7}{|l|}{ Mathematics } \\
\hline No marks & 32 & 1.08 & $0.75-1.56$ & 52 & 1.06 & $0.80-1.40$ \\
\hline Not passed & 765 & 1.11 & $1.01-1.22$ & 219 & 1.21 & $1.06-1.38$ \\
\hline Passed & 1,203 & 1.00 & Reference group & 1,792 & 1.00 & Reference group \\
\hline Passed with distinction or excellence & 931 & 0.89 & $0.82-0.97$ & 1,151 & 0.85 & $0.79-0.92$ \\
\hline \multicolumn{7}{|l|}{ Swedish } \\
\hline No marks & 59 & 1.06 & $0.80-1.37$ & 87 & 1.14 & $0.91-1.43$ \\
\hline Not passed & 684 & 1.04 & $0.95-1.15$ & 151 & 1.34 & $1.13-1.58$ \\
\hline Passed & 1,299 & 1.00 & Reference group & 1,435 & 1.00 & Reference group \\
\hline Passed with distinction or excellence & 919 & 0.78 & $0.71-0.85$ & 1,541 & 0.89 & $0.82-0.96$ \\
\hline \multicolumn{7}{|l|}{ English } \\
\hline No marks & 41 & 1.07 & $0.78-1.47$ & 52 & 1.18 & $0.89-1.56$ \\
\hline Not passed & 587 & 1.19 & $0.90-1.47$ & 176 & 1.24 & $1.06-1.45$ \\
\hline Passed & 1,300 & 1.00 & Reference group & 1,360 & 1.00 & Reference group \\
\hline Passed with distinction or excellence & 1,003 & 0.89 & $0.82-0.97$ & 1,626 & 0.91 & $0.84-0.98$ \\
\hline
\end{tabular}

ORs with $95 \%$ CIs after adjustment for year of birth, maternal age, parity and maternal education 
Fig. 1 Comparison of school marks between children whose mothers had diabetes during pregnancy and all children. Marks for sports (a), mathematics (b), Swedish (c) and English (d) are given as numerical marks (1-5) for 19881997 (left-hand side of each figure part) and as alphabetical marks for 1998 onwards (right-hand side of each figure part). ORs are shown with $95 \%$ CIs (vertical lines) after adjustment for year of birth, maternal age, parity and educational level. 0 , no marks in this subject; 1-5, numerical marks; NP, not passed; P, passed; PD, passed with distinction; PE, passed with excellence

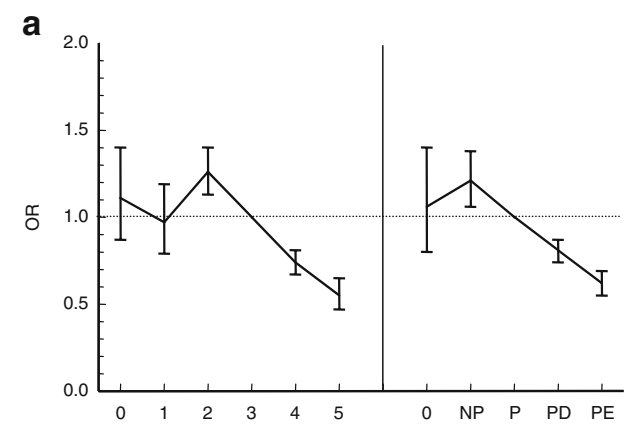

b
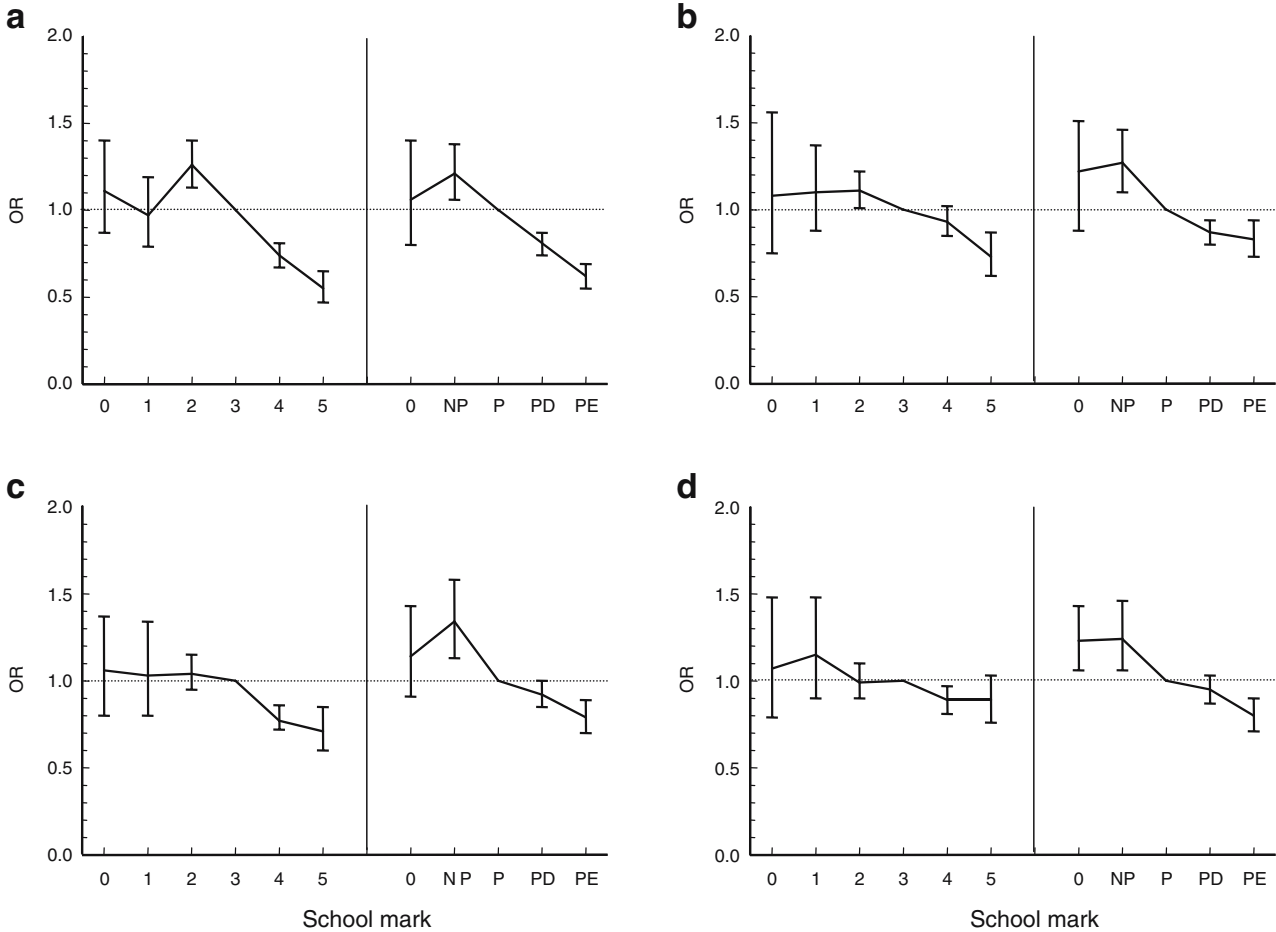

d

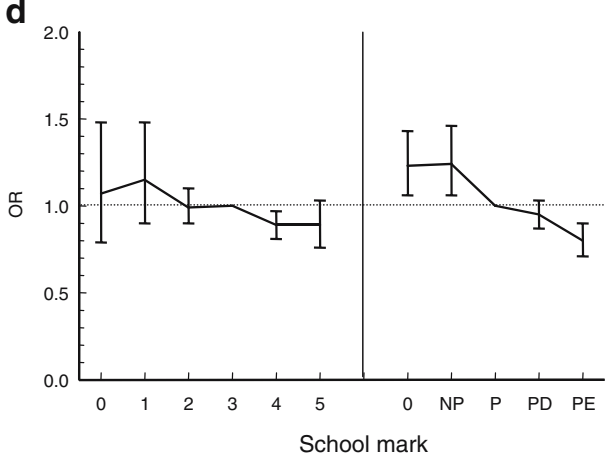

\section{Discussion}

Using a nationwide population-based register study, we show that children born to mothers with diabetes during pregnancy between 1973 and 1986 showed long-term effects with slight but statistically significant lower school performance when leaving compulsory school, usually at the age of 16 years. These effects were seen also when exclusions were made for important perinatal events also associated with diabetic pregnancies, such as high birthweight, prematurity, low Apgar score, malformations or birth by caesarean section. Also multiple pregnancies were excluded. The result indicates that the intrauterine milieu

Table 4 Effect of maternal diabetes on the child's choice of level of mathematics and English language courses (1988-1997): these choices were available only during these years

\begin{tabular}{lllll}
\hline & Common course & Advanced course & OR & $95 \%$ CI \\
\hline English & & & & \\
All & 1,835 & 918 & 1.31 & $1.21-1.43$ \\
Boys & 838 & 560 & 1.36 & $1.22-1.52$ \\
Girls & 997 & 358 & 1.29 & $1.14-1.46$ \\
Mathematics & & & \\
All & 1,467 & 1,317 & 1.30 & $1.20-1.40$ \\
Boys & 737 & 685 & 1.35 & $1.21-1.51$ \\
Girls & 730 & 632 & 1.26 & $1.12-1.40$ \\
\hline
\end{tabular}

ORs are adjusted for year of birth, maternal age, parity and maternal educational level when the mother had diabetes may have had long-term effects on the child's development despite a normal perinatal history. This accords with the findings that in well-controlled gestational diabetes, children of school age were found to have lower verbal IQ scores as well as affected fine and gross motor function compared with controls matched for age, birth order and parental socioeconomic status [13].

Perinatal mortality and morbidity among infants born of diabetic mothers has been clearly associated with a poor metabolic control in the mother. No association could be found between effects on neurological function in school children born to well-controlled diabetic mothers and the medical status of the newborn (high/low birthweight, hypoglycaemia) [14]. This agrees with our finding that exclusion of children with such perinatal complications did not affect the risk increase observed.

A study of children of women with pre-gestational or gestational diabetes found significant associations between low IQ in the child at 2 years and plasma $\beta$-hydroxybutyrate or NEFA levels in the third trimester, whereas no correlations could be found to, for example, indices of glycaemia or perinatal events [7].

A drawback of our register-based study was that we had no access to information on maternal metabolic control during pregnancy. Furthermore, it was not possible to distinguish pre-existing type 1 diabetes from type 2 or gestational diabetes, because before 1987 the classification code used in Sweden (ICD-8) did not differentiate the different types. The 
total rate of identified maternal diabetes was 4.9 per 1,000 , which indicates that the vast majority were type 1 pre-existing diabetes. Misclassification of diabetes diagnosis may exist but would not be associated with the outcome variables and will thus result in an underestimation of risks. The major advantages of a register-based study are that we can use very large data sets and then adjust for a number of potential confounders. Only large data sets may disclose effects on potentially severe complications of low frequency.

It is concluded that despite efforts to improve diabetes care during pregnancy through national healthcare programmes in Sweden, we found low but statistically significant effects on the child's school performance up to the age of 16 years. It should be remembered that for natural reasons, the situation mirrors the therapeutic traditions during the 1970s and 1980s and the effect of further modifications cannot be studied until later on. The effects are moderate and it should be emphasised that the majority of children born to diabetic mothers performed well. However, the findings may suggest that the intrauterine milieu caused by diabetes in the mother may have had long-term effects on the child and call for an improved care of diabetic women during pregnancy. Similar studies from other countries are warranted.

Acknowledgements The study was supported by grants from the Swedish Research Council (Project no. 07531), the Västerbotten County Council (G. Dahlquist) and the K. and A. Wallenberg Foundation (B. Källén).

Duality of interest The authors declare that there is no duality of interest associated with this manuscript.

\section{References}

1. Girlin J, Donhorst A (2003) Pregnancy and diabetes mellitus. In: Pickup JC, Williams G (eds) Textbook of diabetes, vol 2. Blackwell, Oxford, pp 1-39 (chapter 65)
2. Boney CM, Verma A, Tucker R, Vohr BR (2005) Metabolic syndrome in childhood: association with birth weight, maternal obesity, and gestational diabetes mellitus. Pediatrics 115:290-296

3. Silverman BL, Rizzo TA, Cho NH, Metzger BE (1998) Long-term effects of the intrauterine environment. The Northwestern University Diabetes in Pregnancy Centre. Diabetes Care 21(Suppl 2): B142-B149

4. Ornoy A, Wolf A, Ratzon N, Greenbaum C, Dulitzky M (1999) Neurodevelopmental outcome at early school age of children born to mothers with gestational diabetes. Arch Dis Child Fetal Neonatal Ed 81:F10-F14

5. Ornoy A (2005) Growth and neurodevelopmental outcome of children born to mothers with pregestational and gestational diabetes. Pediatr Endocrinol Rev 3:104-113

6. Ornoy A, Ratzon N, Greenbaum C, Wolf A, Dulitzky M (2001) School-age children born to diabetic mothers and to mothers with gestational diabetes exhibit a high rate of inattention and fine and gross motor impairment. J Pediatr Endocrinol Metab 14(Suppl 1):681-689

7. Rizzo T, Metzger BE, Burns WJ, Burns K (1991) Correlations between antepartum maternal metabolism and child intelligence. N Engl J Med 325:911-916

8. National Board of Health and Welfare (1999) National guidelines for care and treatment of diabetes mellitus [in Swedish]. National Board of Health and Welfare, Stockholm, Sweden

9. National Agency for Education (2004) Education resultsnational level. Available at http://www.skolverket.se/sb/d/1637, accessed 13 June 2007

10. Cnattingius S, Ericson A, Gunnarskog J, Källen B (1990) A quality study of a medical birth registry. Scand J Soc Med 18:143-148

11. National Board of Health and Welfare, Stockholm, Sweden (2003) Centre for Epidemiology. The Swedish medical birth register-a summary of content and quality (available at: http://www.sos.se/ FULLTEXT/112/2003-112-3/2003-112-3.pdf, accessed 13 June 2007)

12. Nyström L, Dahlquist G, Rewers M, Wall S (1990) The Swedish childhood diabetes study - analysis of the temporal variation in diabetes incidence 1978-87. Int J Epidemiol 19:141-146

13. Rizzo TA, Dooley SL, Metzger BE, Cho NH, Ogata ES, Silverman BL (1995) Prenatal and perinatal influences on longterm psychomotor development in offspring of diabetic mothers. Am J Obstet Gynecol 173:1753-1758

14. Ornoy A, Ratzon N, Greenbaum C, Peretz E, Soriano D, Dulitzky M (1998) Neurobehaviour of school age children born to diabetic mothers. Arch Dis Child Fetal Neonatal Ed 79:F94-F99 